\title{
Review of "Open Data"
}

\author{
Leonardo Candela ${ }^{1}$ \\ 1 Italian National Res earch Council
}

The definition focuses on Research Data thus it should be better to reconsider the term to be defined from "Open Data" to "Open Research Data".

Regarding Open Data I'm wondering whether what's on Wikipedia is more appropriate here. A suitable definition of Open Data should be something like "Data that are available to everyone to use and republish as they wish, without restrictions from copyright, patents or other mechanisms of control." 